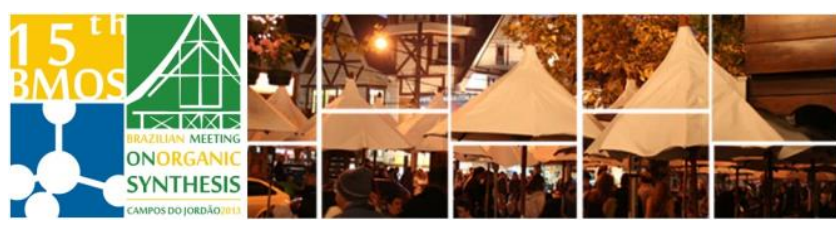

\title{
Biginelli reaction using lemon juice as catalyst under microwave irradiation
}

\author{
Thalita Corrêa C. de Oliveira *(IC) and Andrea Luzia F. de Souza (PQ)
}

\section{Universidade Federal do Rio de Janeiro, Laboratório de Catálise Aplicada em Síntese Orgânica, Campus Macaé Professor Aloisio Teixeira, Avenida Aloizio da Silva Gomes, 50, Granja dos Cavaleiros, Macaé, RJ - Brazil}

*thalitaoliveiraufrj@gmail.com

Keywords: Biginelli, Microwave, Lemon

\section{INTRODUCTION}

Actually, multicomponent reaction (MCR) has been seen target of organic synthetic chemists due your specificity in furnish desired products without side reactions. In 1893, Pietro Biginelli published your pioneer research about a MCR that knowed as Biginelli reactiob. ${ }^{1}$ This MCR was carried out in onepot and furnished 5-ethoxycarbonyl-6-methyl-4phenyl-3,4- dihydropirimidin-2(1H)-one (DHPM) as only product from reaction between benzaldehyde, ethyl acetoacetate and urea under acid catalysis.

Biginelli reaction has been developed in several reaction medium, mainly under microwave irradiation ${ }^{2}$. This reaction has been furnished many compounds with pharmacological activity potentials such as, for example, Monastrol, that can anticancer activity. Many studies shown that DHPMs has been prepared from Biginelli reaction in presence or free-solvent and different catalysts.

In this work was realized a methodology for obtention DHPMs via Biginelli reaction under microwave irradiation, lemon juice was used as catalyst.

\section{RESULTS AND DISCUSSION}

The work was initiated with Biginelli reaction under microwave irradiation (domestic microwave Brastemp - $800 \mathrm{~W})$. The general procedure: aldehyde $(10 \mathrm{mmol})$, ethyl acetoacetate $(10 \mathrm{mmol})$, urea or thiourea $(10 \mathrm{mmol})$ and lemon juice $(0,5 \mathrm{~mL})$ as catalyst (Scheme 1). The results are described in the table 1 . The products were recrystallized by ethanol. All DHPMs was characterized by melting point and compared with literature.

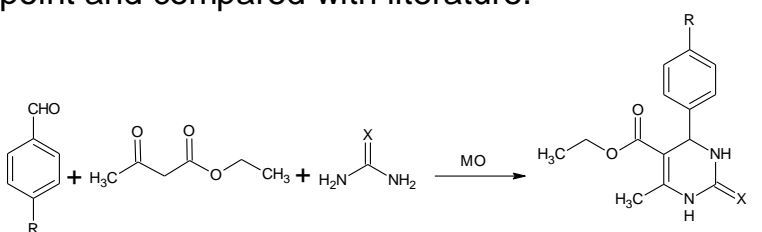

Scheme 1. Biginelli reaction using lemon juice as catalyst
Table 1. Results of Biginelli reaction between aromatic aldehyde, urea or thiourea, ethyl acetoacetate using lemon juice under microwave irradiation.

*Urea **Thiourea

\begin{tabular}{|c|c|c|c|}
\hline Entry & Aldehyde & $\begin{array}{c}\text { Time } \\
\mathbf{( s )}\end{array}$ & $\begin{array}{c}\text { Yield } \\
(\%)\end{array}$ \\
\hline $1^{*}$ & $m$-bromobenzaldehyde & $80-90$ & 80 \\
\hline $2^{*}$ & $m$-fluorobenzaldehyde & $70-80$ & 85 \\
\hline $3^{*}$ & $\begin{array}{c}p \text {-dimethylamino- } \\
\text { benzaldehyde }\end{array}$ & $50-60$ & 92 \\
\hline $4^{*}$ & $p$-nitrobenzaldehyde & $50-60$ & 90 \\
\hline $5^{*}$ & $p$-hydroxybenzaldehyde & $60-70$ & 83 \\
\hline $6^{*}$ & $m$-hydroxybenzaldehyde & $60-70$ & 80 \\
\hline $7^{*}$ & Benzaldehyde & $60-70$ & 65 \\
\hline $8^{*}$ & o-hydroxybenzaldehyde & $60-70$ & 78 \\
\hline $9^{* *}$ & o-hydroxybenzaldehyde & $50-60$ & 72 \\
\hline $10^{* *}$ & $m$-hydroxybenzaldehyde & $60-70$ & 75 \\
\hline $11^{* *}$ & $p$-hydroxybenzaldehyde & $70-80$ & 81 \\
\hline $12^{* *}$ & $\begin{array}{c}p \text {-dimethylamino- } \\
\text { benzaldehyde }\end{array}$ & $60-70$ & 89 \\
\hline $13^{* *}$ & $p$-nitrobenzaldehyde & $50-60$ & 93 \\
\hline \multicolumn{3}{|c|}{} \\
\hline
\end{tabular}

The results were satisfactory compared to different aldehydes and urea/thiourea. All melting points of DHPMS were compared with literature.

\section{CONCLUSION}

DHPMs were successfully provided in a simple system under microwave using lemon juice as a catalyst. This "green" system was applied in different aldehydes to prove its efficiency. This system can be used, for example, to experimental classes about multicomponent reaction and green chemistry.

\section{ACKNOWLEDGEMENTS}

CNPq, Faperj

\section{REFERENCES}

Biginelli, P. Gaz. Chim. Ital. 1893, 23, 360.

${ }^{2}$ Sandhu, J. S. Arkivoc 2012, (i), 66. 